\title{
Analyses of the inferior turbinate using 3D geometric morphometrics: an anatomical study and discussion of the potential clinical implications*
} \author{
F. Savall ${ }^{3,4}$ \\ Otorhinolaryngology, Head and Neck Surgery Department, University Hospital Rangueil-Larrey, Toulouse, France \\ ${ }^{2}$ Clinical Anatomy Department, University Hospital Toulouse-Rangueil, Toulouse, France \\ ${ }^{3}$ National Committee for Scientific Research (CNRS), Research Unit 5288, Paul Sabatier University, Toulouse III, France \\ ${ }^{4}$ Legal medicine department, University Hospital Toulouse-Rangueil, Toulouse, France
}

\begin{abstract}
Background: The inferior turbinate is probably the most important anatomical structure within the nasal airway. However, relatively little is known concerning its 3D anatomy. Complete removal of the IT may lead to severe functional disorders and reconstruction is often necessary.
\end{abstract}

Methods: We performed an anatomical study using modern 3D reconstruction tools (Amira 5.4.2 software). The study was conducted on 200 inferior turbinates obtained from CT-scanned images. Metric and morphological analyses were performed. Our aim was to assess 3D polymorphism of the inferior turbinate in humans, and to evaluate gender dimorphism and age-related differences.

Results: Anatomical characteristics of the inferior turbinate were poorly heterogeneous. Metric analyses revealed no gender dimorphism or age-related differences, except in the elderly, where the inferior turbinate was usually less curved. Morphological analyses defined that the mean shape was related to age and gender. Differences were limited and are summarized in this manuscript.

Conclusions: This very reproducible methodology may have clinical applications regarding, e.g., sinonasal cancer or empty-nose syndrome.

Key words: inferior turbinate, anatomy, gender dimorphism, age-related differences, metric analysis

\section{Introduction}

The inferior turbinate (IT) is a bone within the lateral wall of the nasal cavity. It is probably one of the most important structures within sinonasal physiology. Its key-role is well documented but has been underestimated. Complete removal of the IT may lead to severe functional disorders; airflow in the nasal cavity is modified, and there is loss of turbulence. Zhao et al. have described airflow disruption after inferior turbinate resection in a computational model of nasal-airflow dynamics ${ }^{(1)}$. According to this model, loss of turbinate tissue disrupts the structure of the inferior meatus, leading to turbulent, less efficient, and poorly perceived airflow. In this context, several authors have argued for the need for IT reconstruction for three main diagnoses: i.e., atrophic rhinitis ${ }^{(2)}$, empty nose syndrome ${ }^{(3)}$, and the functional consequences after medial maxillectomy ${ }^{(4)}$. Few publications have reported on restoring IT anatomy to increase patients' quality-of-life. It is important to assess and understand the complex anatomy of the IT. Studies that focus on the IT anatomy are rare, and only focus on elementary distances and angles. No study has used a 3D multi-dimensional approach. In recent years, $3 \mathrm{D}$-imaging systems have become widely available. A metric approach with rigorous and reproducible methodology using 
3D reconstructions, obtained from computed-tomography (CT) mages, have become more objective than morphological descriptions of bone through visual analyses. The main advantage feometric mophometics over tradition morphological (a) 列 cally preserved and is statistically interpretable ${ }^{(5)}$. Geometric morphometric analyses combine the powerful and flexible tools from multivariate statistics, thus making investigation into morphological variations possible, and provide direct reference to the anatomical structure studied( $)$. Geometric morphometric analysis has been developed to quantify the shape of rigid structures that have curves and bulges, and that are not easily alyzed by traditiona metric methods. Our study focused on (n) cann be considered as a rigid structure and varies widely during the nasal cycle ${ }^{(7)}$.

The aim of our study was i) to assess 3D polymorphism of the IT in humans, ii) to evaluate gender dimorphism and age-related differences, and iii) to discuss the potential applications to fore sic science and clinical practice.

\section{Methods}

Ethics approval and consent to participate: According to French law, the results from medical imaging could be used retrospec tively without the patient's consent when the examinations were carried out for clinical purposes and when the results were recorded anonymously (article 40-1, law 94-548 of 1 July 1994).

Sample

We carried out a retrospective study of the inferior turbinate within individuals undergoing clinical multislice computed tomography (MSCT) between 2013 and 2016. The MSCT examinations were mainly requested within the clinical context of chronic headaches. Patients with a known history of sino-nasal complaints or that had opacities on at least one sinus cavity were excluded. Only scans that showed both the entire inferior turbnate, the hard palate, and the anterior skull base were retained. The patients were of various ethnic origins and were globally representative of the present-day population of south-western France. The data and images were recorded anonymously with only gender and age recorded at the time of the CT.

Multislice computed tomography

The MSCT images were obtained through a Picture Archiving and Communication System (PACS, McKesson Medical Imaging Group, Richmond, BC, Canada). Examinations were performed on a Sensation 16 Scanner (Siemens, Erlangen, Germany) with 16*1.5-mm collimation. The image matrix was 512×512 pixels. A bone filter was used. Depending on the purpose of the examination, axial reconstructions were performed at 1-mm intervals. Scans were saved as digital images and communications-in-me

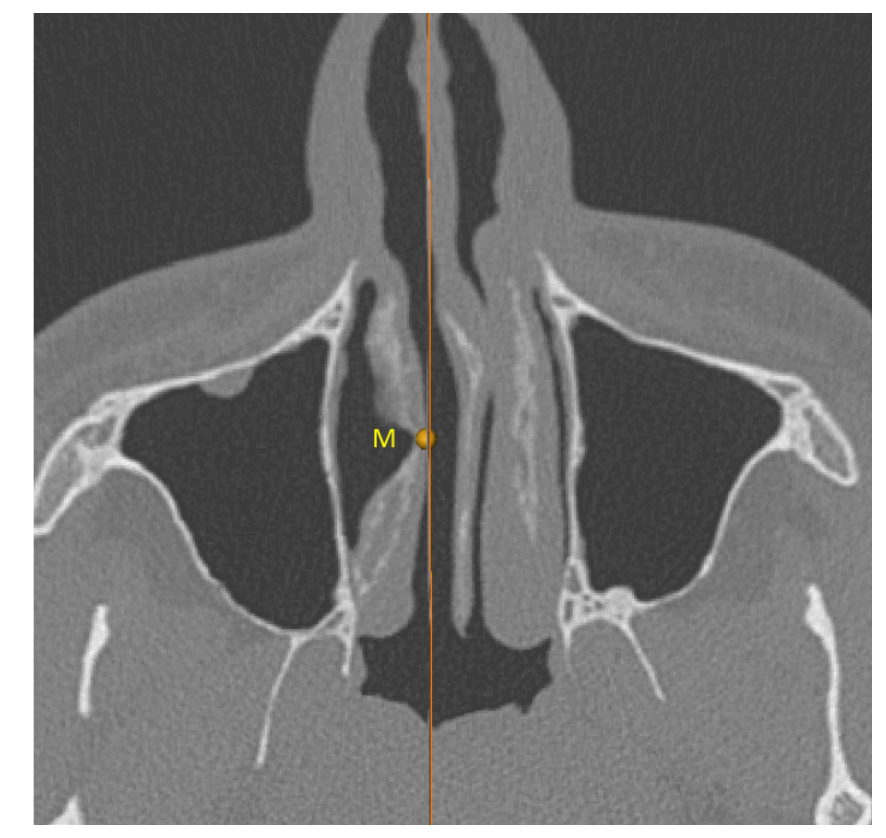

Table 1. Anatomical description of the landmarks used.

\begin{tabular}{ccc}
$\begin{array}{c}\text { Land- } \\
\text { marks }\end{array}$ & $\begin{array}{c}\text { Type of } \\
\text { landmark }\end{array}$ & \multicolumn{1}{c}{ Anatomical definition } \\
\hline M & II & $\begin{array}{l}\text { The most medial point of the inferior turbi- } \\
\text { nate (checked in } 3 \text { planes) }\end{array}$ \\
A & II & $\begin{array}{l}\text { The anterior point located at the level of the } \\
\text { piriform aperture }\end{array}$ \\
P & II & $\begin{array}{l}\text { The most posterior point of the inferior } \\
\text { turbinate }\end{array}$ \\
S & III & $\begin{array}{l}\text { The most superior point of the inferior } \\
\text { turbinate located in the same cutting plane } \\
\text { as the medial point }(\mathrm{M})\end{array}$ \\
I & III & $\begin{array}{l}\text { The most inferior point of the inferior tur- } \\
\text { binate located in the same cutting plane as } \\
\text { the medial point }(\mathrm{M})\end{array}$ \\
\hline
\end{tabular}

viation for each subgroup. Comparative analyses were performed with all the variables based on gender (male, female) and age. Three sub-groups ( $A, B$ and $C$ ) were defined according to patient's age. The normal distribution of the data was tested using the Shapiro-Wilk's test. Sexual dimorphism for each metric parameter was compared using parametric (student test) or non parametric (Mann-Whitney test) tests. Group age-related differences were assessed using parametric (analysis of variance (ANOVA)) or non parametric (Kruskal-Wallis) test. Classical linear regression was used between metric parameter and age and in order to bring more precision, we illustrated this regression using the "broken line" function (R-3.3.2 - Package 'segmented') for the antero-inferior angle. This method allowed us to create a segmented model and compute the fitted values for each segmented relationship ${ }^{(10)}$.

Morphological analyses

A generalized Procrustes analysis was carried out on the 3D coordinates. As described previously, this strategy expresses the results in a graphical format by showing the average shape of the subgroups of interest. The landmark coordinates were analyzed using principal-component analysis and canonical variate analysis to identify the shape trends in the various subgroups ${ }^{(1)}$. Discriminant analysis was performed on CVscores to determine the percentage of cases in which gender was correctly estimated. To determine if the difference between shapes was statistically significant, a P-value was calculated using Goodall's F-test and Mahalanobis D2 matrices ${ }^{12}$.

\section{Results}

Reliability of the landmark locations

The G-coefficient was very close to 1 ( 0.97 for intra- and 0.93 for inter-rater): thus, the level of agreement on landmark locations between the two raters was high. Consequently, we concluded on the reliability of the landmark locations. Furthermore, the va-

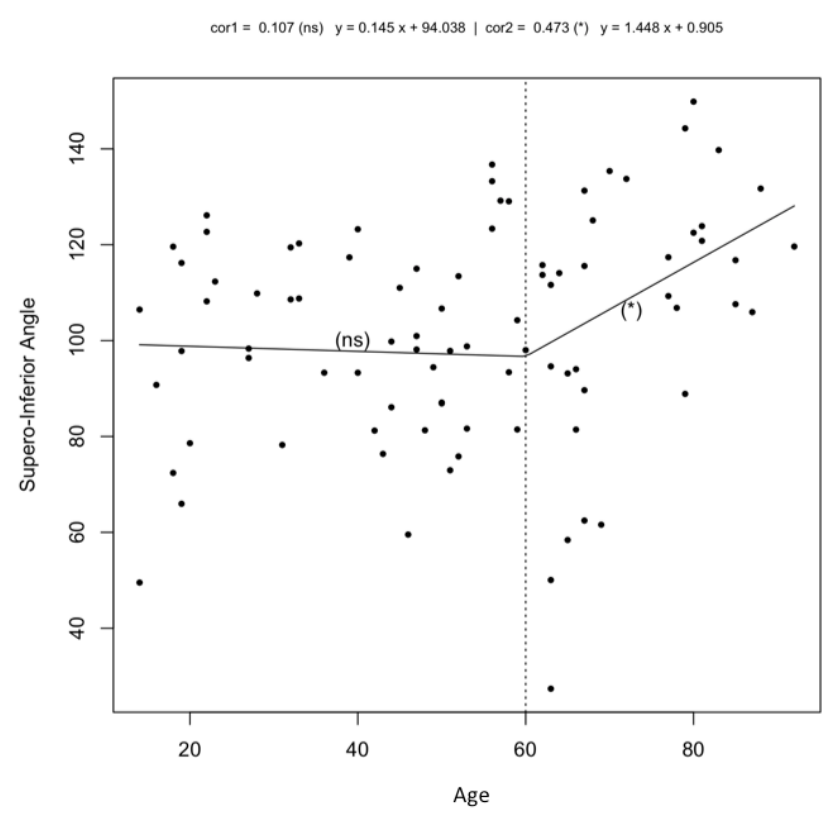

Figure 2. Linear regression of superior-inferior angle, depending on age * Linear regression revealed statistical evidence after 60 years.

riance components used to calculate the $\mathrm{G}$ coefficient provided the following details. It was apparent that the greatest source of variability was the landmark (38.8\% for intra- and $42.13 \%$ for inter-rater). The rater and landmark-interaction variance ratios were very low $(0.23 \%$ for intra- and $4.1 \%$ for inter-rater). Another source of variability was the landmark and subject interactions. The explained variance ratio for this variability was not good ( $24.48 \%$ for intra- and 27.26 for inter-rater).

\section{Metric analyses}

A total of $100 \mathrm{CT}$ examinations from individuals were included: 48 women and $52 \mathrm{men}$. Their mean age was 52 years (minimum age $=14$ years, maximum age $=88$ years). Number of patients in each sub-group was nearly equivalent ( 32 in group $A, 38$ in group $B$ and 30 in group C). Descriptive statistics for anthrometric measurements according to gender and age are given in Table 2. Concerning the metric analyses of the IT, we found no difference between gender, side, or age. For the superior-inferio angle (normal distribution), ANOVA revealed a statistically significant difference between groups A $(<30 y)$, B (between 30 and 60 years old), and $C$ (up to $60 y$ ) (Fvalue $=4.4, p$-value $=0.015$ ). Student t test revealed significant difference between group $A$ and group $C(p$ value $=0.02)$ and between group $B$ and $C(p$ value $=0.01$ ). There is not significant difference between group $A$ and group $B(p=0.55)$

The same was true for the antero-posterior angle without reaching any statistical evidence. Both angles tended to widen in age. The positivity of the Pearson's correlation coefficient indicates that the angle increased in elderly patients: this was particularly true for those aged $>60$ years (Figure 2, linear regres- 
Table 2. Mean values ( $\min$ - max: SD) of the anthropometric variables based on gender and age.

\begin{tabular}{|c|c|c|c|c|c|c|c|}
\hline & AM & MP & AP & SM & SI & $\begin{array}{l}\text { Superior-inferi- } \\
\text { or angle }\end{array}$ & $\begin{array}{l}\text { Antero-posteri- } \\
\text { or angle }\end{array}$ \\
\hline Female & $\begin{array}{c}18.8 \\
(10 ; 28) 4\end{array}$ & $\begin{array}{c}18.1 \\
(9 ; 27) 3\end{array}$ & $\begin{array}{c}35 \\
(24 ; 43) 5\end{array}$ & $\begin{array}{c}10.6 \\
(7.4 ; 20) 3\end{array}$ & $\begin{array}{c}15.9 \\
(8 ; 27) 4\end{array}$ & $\begin{array}{c}98 \\
(30 ; 150) 26\end{array}$ & $\begin{array}{c}144.3 \\
(95 ; 164) 10\end{array}$ \\
\hline Male & $\begin{array}{c}18.5 \\
(10 ; 29) 4\end{array}$ & $\begin{array}{c}18.8 \\
(5 ; 30) 5\end{array}$ & $\begin{array}{c}35 \\
(20 ; 46) 7\end{array}$ & $\begin{array}{l}10.7 \\
(6 ; 23) 3\end{array}$ & $\begin{array}{c}17.4 \\
(7 ; 31) 5\end{array}$ & $\begin{array}{c}105 \\
(60 ; 144) 20\end{array}$ & $\begin{array}{c}139.8 \\
(44 ; 163) 19\end{array}$ \\
\hline $\begin{array}{l}\text { Age }<30 \mathrm{yr} \\
\text { (group A) }\end{array}$ & $\begin{array}{c}18.4 \\
(10 ; 25) 4\end{array}$ & $\begin{array}{c}19 \\
(11 ; 23) 4\end{array}$ & $\begin{array}{c}35.5 \\
(20 ; 43.6) 6.2\end{array}$ & $\begin{array}{c}10.2 \\
(5.7 ; 16.2) \\
2.3\end{array}$ & $\begin{array}{c}17.4 \\
(8,28) 5.1\end{array}$ & $\begin{array}{c}99.6 \\
(49.5 ; 126) 19\end{array}$ & $\begin{array}{c}145.1 \\
(128 ; 163) 7\end{array}$ \\
\hline $\begin{array}{l}\text { Aged } 30 \text { - } 60 \mathrm{yr} \\
\text { (group B) }\end{array}$ & $\begin{array}{c}18.8 \\
(13 ; 28) 3.7\end{array}$ & $\begin{array}{c}22 \\
(15 ; 30) \\
3.2\end{array}$ & $\begin{array}{c}35 \\
(24.4 ; 45.8) \\
4.9\end{array}$ & $\begin{array}{c}10.8 \\
(6.1 ; 23) \\
3.8\end{array}$ & $\begin{array}{c}15.7 \\
(6,30) \\
4.9\end{array}$ & $\begin{array}{c}96.2 \\
(56 ; 136) 20\end{array}$ & $\begin{array}{c}140.8 \\
(84 ; 156) 10\end{array}$ \\
\hline $\begin{array}{l}\text { Aged }>60 \text { yr } \\
\text { (group C) }\end{array}$ & $\begin{array}{c}18.4 \\
(9.7 ; 39) \\
4.8\end{array}$ & $\begin{array}{c}20.1 \\
(12 ; 22) 3\end{array}$ & $\begin{array}{c}34.1 \\
(20.6 ; 46) 7.2\end{array}$ & $\begin{array}{c}10.8 \\
(7.9 ; 17) 2\end{array}$ & $\begin{array}{c}17.1 \\
(11,22) 31\end{array}$ & $\begin{array}{c}113.4 \\
(61 ; 149)\end{array}$ & $\begin{array}{c}139.7 \\
(56 ; 164) 23\end{array}$ \\
\hline
\end{tabular}

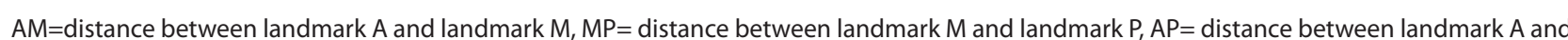
landmark $\mathrm{P}, \mathrm{SM}=$ distance between landmark $\mathrm{S}$ and landmark $\mathrm{M}, \mathrm{S} \mathrm{S}=$ distance between landmark $\mathrm{S}$ and landmark $\mathrm{L}$

sion lines using broken-line function)

\section{Morphological analyses}

Morphological analyses revealed gender- and age-related dimorphism. The shapes of the male and femalenferior turbinates are shown in Figure 3. In the 3D graphic ion therewere and ages, and 3 geos and ages, and 3D geometric analyses defined an asymmetric triangular area with a medial point located more posteriorly and laterally in elderly patients and in women (Figure 3). The posterior landmarks of women were higher than those for men, the anterior landmarks were more posterior than those of men and the superior landmark more posterior, lower, and externa all's $F$ and the Mahalanobis distance values indicated that the male and female inferior turbinate differed in shape (Mahalanobis D2 distance $=1.17$, Goodall's F $=0.83, P=0.04)$. PCA of the IT represented $41 \%$ of the explaine variance for sex and age. The CVA revealed that the correct sex was assigned in $70 \%$ of cases and the cross-validation revealed $75 \%$ rate of correct sex estimation (Figure 4 ).

\section{Discussion}

The study, we have shown that the characteristics of the IT, as defined by metric analyses and geometric morphometrics, including gender and age, were very heterogeneous and were only linked with advanced age. Variations in 3D morphology were few according to gender and age. Morphometric geometrics analyses focus on shape differences related to sex and age and provide additional informations. With Generalized Procrustes Analyses, size effects related to isometry were removed but allometric size differences were retained and visible.

Few anthropometrics and anatomical factors seem to influence IT morphology, probably because the IT plays a key-role in nas airflow and slight variations would modify airflow and, thus, a patient's comfort. The most important modifications appeared in elderly patients. These patients had decreased IT curvature, with wider angles, and their ITs were globally smaller, probably because of the loss in osteous density.

Comparison with others studies

There are limited published data concerning the surgical anatomy of turbinates, even though this is a very common issue in routine clinical practice. Data concerning dimensions of the IT are sparse and do not distinguish between mucosal and bone morphologies. We have focused on the anatomy of the IT bone nd have not considered the mucosal part of the IT because it is very difficult to assess. The impact on nasal cycles or problems Whe as chronic rhinitis, could not be fully explored in this study. We also only focused on ma bones, the deve during the first decade will be developed in a future publication.

Balbach et al. ${ }^{(13)}$ recently used digital-volume tomography to assess the length and height of the IT. Their results are summarized as follows. The bony length of the inferior turbinate in the antero-posterior direction was $38.9+4.0 \mathrm{~mm}$ the turbinates in varied between 3.9 and $20.8 \mathrm{~mm}$, bony thickness varied between 0.9 and $2.7 \mathrm{~mm}$. They also assessed the insertion angle of the inferior turbinate in relation to the lateral nasal wall. This angle varied between $57.0^{\circ}$ and $84.2^{\circ}$. These were interesting results but did not provide information concerning dimorphism or age/gender-related differences, and the methodology was unclear. When it comes to dimorphism, we did not report any difference between left and right IT in two studies, Lang et a study $y^{(14)}$ the rightir s longer than the left and there were no significant differences between the left and right sides in the

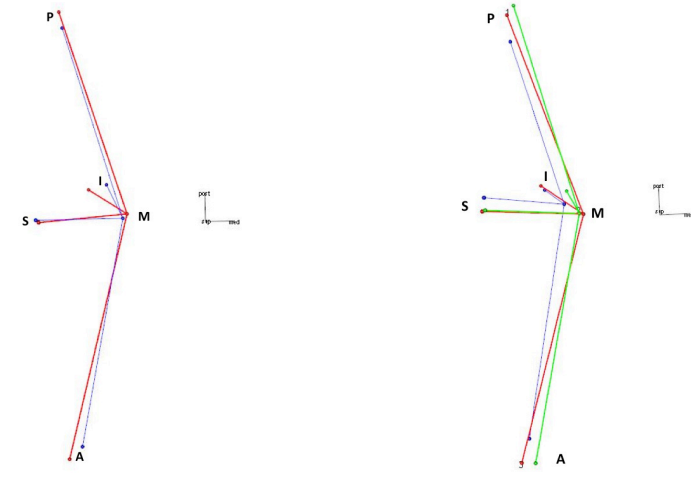

Figure 3. Shape variation based on gender. a) reconstruction of the average for females=blue, males=red) and age. b), reconstruction of the average for patient's age $<30 y=b l u e$, patient's age between 30 and 60 $y=$ red, patient's age $>60 \mathrm{y}=$ green) according to superior view $3 D$.

second study ${ }^{(15)}$. In our analyses, bony length was $39.0 \pm 4.0 \mathrm{~mm}$. No significant differences between male and female patients

has been reported. The only authors that have suggested that the inferior turbinate might differ according to gender and age were San and colleagues(16). They assessed the IT and reported it was significantly smaller in females.

\section{Clinical applicability}

The main clinical applicability of our study was to define the ideal dimensions for custom-made implants, especially in the context of empty-nose syndrome (ENS) or atrophic rhinitis (Figure 5). ENS is a rare iatrogenic disorder that occurs after complete
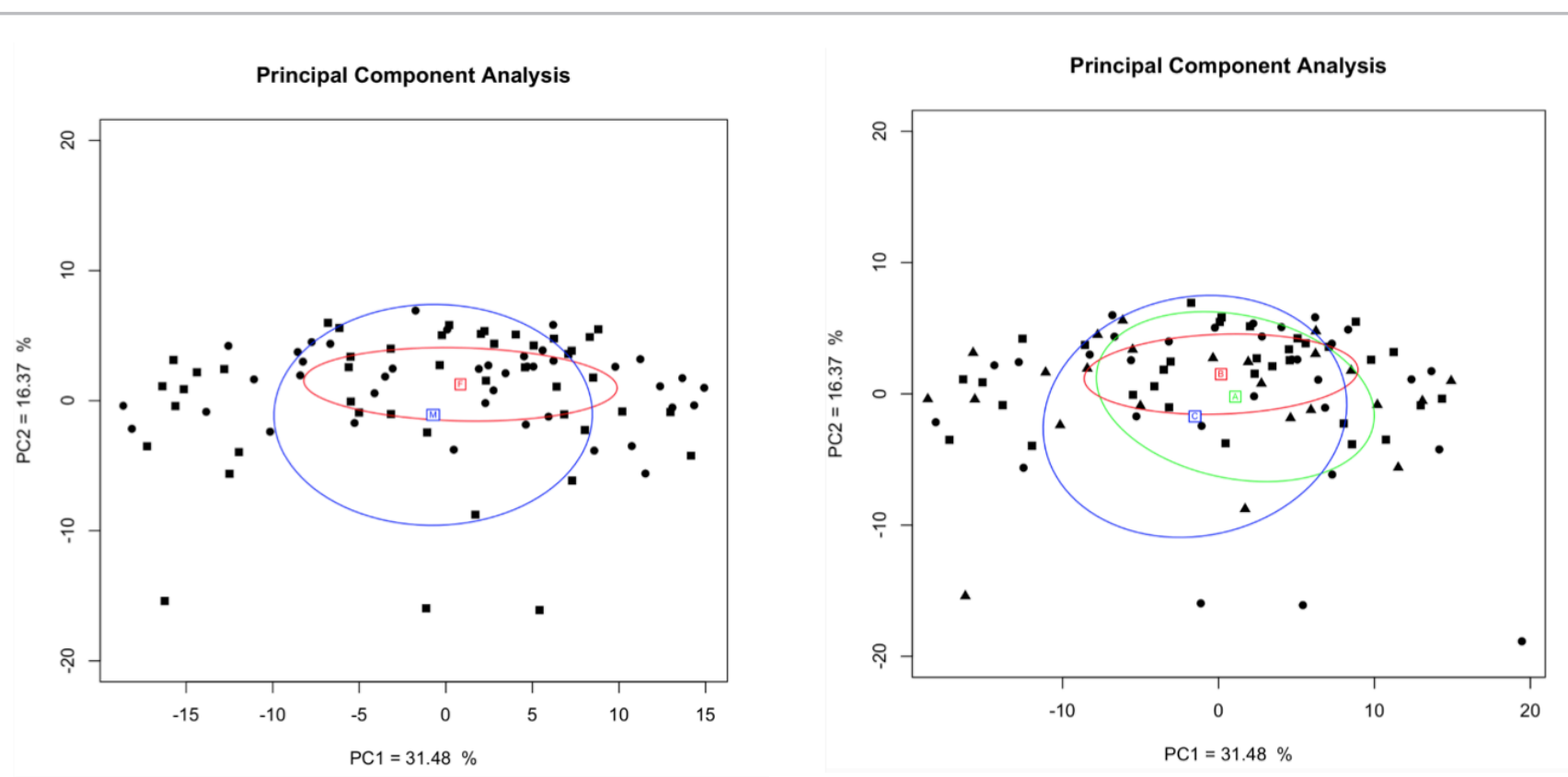

Figure 4 . Principal-component analysis on the shape of the inferior turbinate based on a) gender (females=red, males=blue) and b) age (patient's age $\langle 30 \mathrm{y}=$ blue, patient's age between 30 and $60 \mathrm{y}=\mathrm{red}$, patient's age > $60 \mathrm{y}=$ green). The ellipses correspond to $68 \%$ confidence intervals.

IT resection. Some patients require surgical repair, where the goal is to reduce the empty space by creating a turbinate-like mass with submucosal implantation. Velasquez et al. ${ }^{(17)}$ performed inferior turbinate reconstruction using a porcine smallintestine submucosal xenograft in three patients. Functional outcomes estimated with the SNOT-25 score, showed significan improvement after a 12-week follow-up, SNOT-25 decreased from 77 to 55 . Bastier et al. ${ }^{(18)}$ reported similar results: they used a b-tricalcium phosphate implant in 14 patients. Quality-of-life (frequency, bothersomeness, impact) significantly improved af ter surgery. Other studies have used various materials, including bone, cartilage, muscle, fascia, fat, and also biomaterials ${ }^{(19,20)}$ Despite these interesting results, patients' complaints have remained and further studies to improve anatomic restoration are necessary. Some author ${ }^{(2)}$ emphasize the role of turbinate hypertrophy in nasal obstruction or sinus disease. This study also provides some insights concerning what is really a hypertrophic inferior turbinate (in the bony part): some authors have emphasized the role of bony hypertrophy in patients with IT enlargement and highlighted the need to distinguish it from a mucosal hyperplasia(22). In patients with bony hypertrophy resection of the turbinal bone limited to the inferior part can be proposed whereas in other cases, radiofrequency could be performed. Overall, we note that the thickness of the IT was very thin $(<2 \mathrm{~mm})$. In addition, no standard criteria are available to design the optimal anatomical shape of the turbinate as a target for surgery. A decision between a basal or medial resection can be challenging: previous studies ${ }^{(23)}$ report that basal resection better preserves calculated humidification efficiency. Our re-

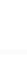




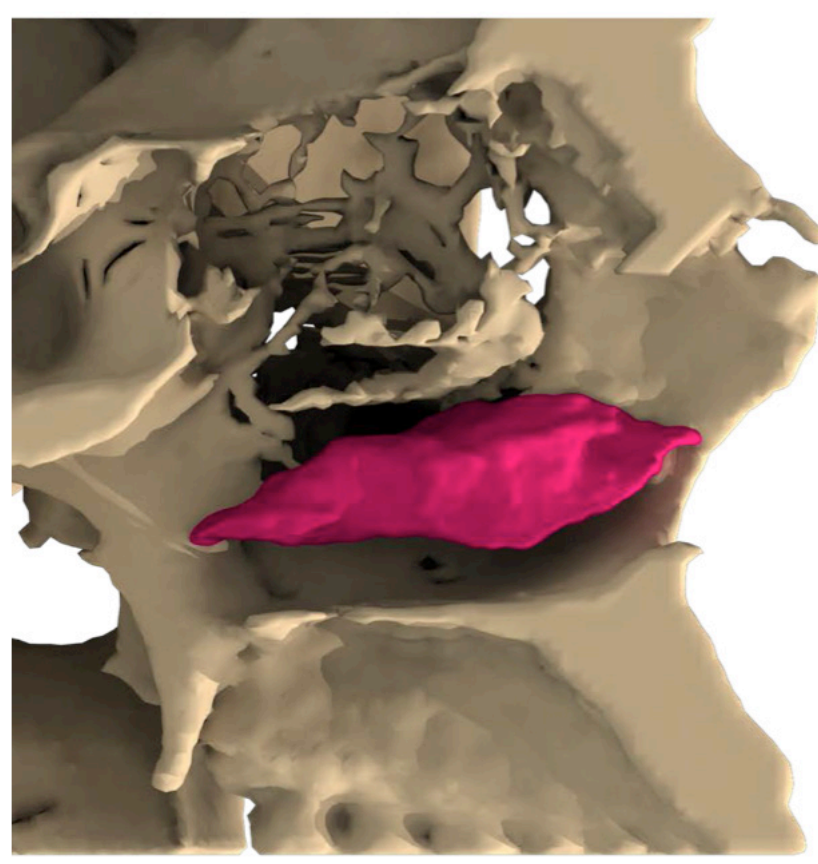

tary dimensions of the IT using a very reproducible method. We are also the first to assess the 3D morphology of the IT in humans. We think this is a fundamental prerequisite to improve functional results after surgical repair. We have also highlighted the with age and gender, and have suggested some possible applcations. Weaknesses of this study include the absence of a true mucosal analysis and the limited interest of the IT anatomy in forensic medicine. Furthermore we did not take septal deviation into account. Further studies are ongoing on these subjects.

\section{Conclusion}

The geometric morphometrics used in our model distinguishes the form of an object from its shape by scaling to a unit size. This makes it possible to model morphological variations without taking size into account. Conformation of the IT exhibits few variations. Right and left IT did not differ significantly. These low variations may reflect the "conserved" 3D anatomy found in the IT with regards to sino-nasal function and nasal airflow.

Figure 5. 3D reconstruction of average shape for women inferior turbnate between 30 and 60 years (medial view)

sults may help in difficult cases and aid surgeons when choosing between the different techniques, including out-fracture.

Strengths/weaknesses of this study

This study is original in many aspects: firstly, we analyzed the

\section{Authorship contribution}

Concept and design: G de Bonnecaze, N Telmon, F Savall

Analysis and interpretation of data: $\mathrm{G}$ de Bonnecaze, $\mathrm{F}$ Savall

Drafting manuscript or revision: All authors.

\section{Conflict of interest}

All authors declare that they have no conflict of interest or

funding. Arch Otorhinotaryngol 2013 Feb; $270(2)$

20. Jiang C, Shi R, Sun Y. Study of inferior tur-
binate reconstruction with Medpor for the treatment of empty nose syndrome May: 123(5): $1106-$

21. Ural A. Songu M Adibelli ZH, Candan H A stereological study on the correlation of inferior turbinate hypertrophy and parana. 22.74 .

nate entergement and thonic inferior turbsurgical intervention. Rhinology. 2006 Deci 44(4):234-8.

23. Harriri BM, Rhee JS, Garcia GJM. Identifying patients who may benefit from inferior turtions. Laryngoscope. 2015 Dec; 125(12) 2635-2641.
DrGuillaume de Bonnecaze MD, PhD Otorhinolaryngology Head and Neck Surgery Department University Hospital Toulouse Rangueil-Larrey Hospital

24 Chemin de Pouvourville

31059 Toulouse

France

Tel: $+33-567771788$ Mobile: $+33+651472078$ Fax: +33-567 771473 E-mail:

debonnecaze.g@chu-toulouse.fr

\section{References}

What is normal nasal airlow? A computational study of 22 healthy (6): $435-446$.

Park MJ, Jang YJ. Successful management of
primary atrophic rhinitis by turbinate reconstruction using autologous costal cartilage. Auris Nasus Larynx. 2018 Jun:45(3):613-616,
Coste A. Dessi P. Serrano E Empty syndrome Eur Ann Otorhinola moly nose Neck Dis. 2012 Apr; 129(2): 93-97.

4. Costa ML, Psaltis AJ, Nayak JV, Hwang PH. Long-term outcomes of endoscopic maxillary mega-antrostomy for refractory chronic 2015 Jan: $5(1) \cdot 60-5$ modern huy the regional variation Phys. Anthropal 2002 lanacial form. An 6. Klingenberg $C P$. Morphometrics and the D. evolution of developmental mechanisms. Gene. 2002 Apr 3; $287(1-2): 3-10$

Pendolino AL, Nardello E, Lund VJ, Maculan between unilateral PNIF and rhinoma- nometry in the evaluation of nasal cycle. Rhinology. 2017 Oct; ahead of print. Cramon-Taubadel von N, Frazier BC, Lahr error in grometric morphometrics theory methods, and modifications. Am J. Phys. Anthropol. 2007 Sep; 134(1): 24-35.

Klingenberg CP. MorphoJ: an integrated software package for geometric morpho-

Muggeo VMR.2008. Segmented: an R ackage to fit regression models with broken-line relationships. R News 8:20-25. A Dimensional Shape Analysis, PLoS One. 2016; 11(3)

12. Oettlé AC, Pretorius E, Steyn M. Geometric morphometric analysis of the use of mantion Homo 2009:60(1): 20.43.

S. Teymoortash A, Werner JA, et al. Radiological examinations of the anatomy of the inferior turbinate using digital volun: 49(2): 248-252.
14. Lang J, Baumeister R. [Postnatal growth of the nasal cavity]. Gegenbaurs Morpho Jahrb. 1982; 128(3): $354-393$.

政 the size and arrangement of the conchae nasales and apertura sinus sphenoidalis (author's trans))]. Anat Anz. 1981; 149(4):

16. San T, Muluk NB, Saylisoy S, Acar M, Cingi sizes differ in subjects grouned by sex and age. Rhinology. 2014 Sep; 52(3): $231-237$. 17. Velasquez $N$, Huang $Z$, Humphreys IM, using porcine small intestine subsuction xenograft demonstrates improved qualty of life outcomes in patients with empty nose syndrome. Int Forum Allergy Rhinol. 2015 Nov; $5(11): 1077-1081$

18. Bastier P-L, Fierens S, Champel S,
Ribadeau-Dumas A, de Gabory L B-Tricat cium Phosphate Implants in the Surgical Treatment of Empty Nose Syndrome. Otolaryngol Head Neck Surg. 2016 Sep; 155(3). 514-517.

grafts versus silastic sheets implants for 